\title{
Multilinguales
}

\section{Les actualisateurs de généricité de la nomination ethnique dans le récit viatique}

Ethnic nomination actualizers of the genericity in the travel literature

$$
\text { المُحيّنات الأجناسية للتعيين الإثني في أدب الرحلة }
$$

\section{Esfandiar Esfandi et Afsaneh Pourmazaheri}

\section{OpenEdition}

\section{Journals}

Édition électronique

URL : http://journals.openedition.org/multilinguales/622

DOI : $10.4000 /$ multilinguales. 622

ISSN : 2335-1853

\section{Éditeur}

Université Abderrahmane Mira - Bejaia

\section{Référence électronique}

Esfandiar Esfandi et Afsaneh Pourmazaheri, «Les actualisateurs de généricité de la nomination ethnique dans le récit viatique », Multilinguales [En ligne], 7 | 2016, mis en ligne le 31 décembre 2016, consulté le 17 septembre 2019. URL : http://journals.openedition.org/multilinguales/622 ; DOI : 10.4000/multilinguales.622

Ce document a été généré automatiquement le 17 septembre 2019.

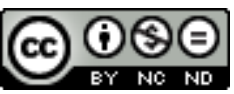

Multilinguales est mise à disposition selon les termes de la Licence Creative Commons Attribution Pas d'Utilisation Commerciale - Pas de Modification 4.0 International 


\section{Les actualisateurs de généricité de la nomination ethnique dans le récit viatique}

Ethnic nomination actualizers of the genericity in the travel literature

$$
\text { المُحيّنات الأجناسية للتعيين الإثني في أدب الرحلة }
$$

\section{Esfandiar Esfandi et Afsaneh Pourmazaheri}

1 Ce travail de recherche interroge le discours de représentation de l'ethnotype "Persan ", à travers certains éléments discursifs qui filtrent son univers référentiel, dans cinq (5) récits de voyageurs français du début du $19^{\mathrm{e}}$ siècle: A. Gardane (De) : Journal d'un voyage dans la Turquie d'Asie et la Perse (1808), J. J. Morier: Second voyage en Perse, en Arménie et dans l'Asie-mineure fait de 1810 à 1816 (1818), J. M. Tancoigne : Lettres sur la Perse et la Turquie d'Asie (1819), P. A. E. P. Jaubert: Voyage en Arménie et en Perse, effectué en 1805-1806 (1821) et G. Drouville : Voyage en Perse fait en 1812-1813 (1828).

2 Notre but est de montrer que le mode de représentation de l'Autre, dans les récits viatiques, passe par un processus linguistique de généralisation et d'homogénéisation du référent pour (re)produire des stéréotypes. La description du référent de l'Autre se réalise, en effet, à travers des constructions dont l'instance énonciatrice est diffuse et le sens implicite.

3 Pour étayer notre hypothèse, nous avons choisi d'analyser et de comparer ces récits parce qu'ils relèvent du même genre, viatique, traitent du même sujet, la Perse et les Persans, et couvrent le premier quart du $19^{\mathrm{e}}$ siècle (1808-1828).

Nous y observerons le traitement de certains actualisateurs de généricité, notamment les articles définis « le » et « les » et l'article indéfini « un », dans des énoncés qui, sous couvert de description de la rencontre nouvelle de l'Autre, mettent en œuvre des structures doxiques, des préjugés et des lieux communs que les récits activent ou réactivent de l'un à l'autre.

5 Cependant, les nombreuses désignations du stéréotype (stéréotype, cliché, poncif, idée reçue, lieu commun, ...) et ses non moins nombreuses définitions rendent nécessaire un 
bref rappel théorique de la notion telle que l'entendent certains théoriciens, avant d'entamer l'analyse proprement dite des actualisateurs de la généralisation de notre corpus.

\section{De quelques repères théoriques de la stéréotypie}

6 Le stéréotype est considéré, du point de vue sémantique, «(...) comme du déjà dit faisant partie de la mémoire discursive de l'énonciateur et structurant son inscription en discours en tant que sujet social » (Détrie et al., 2000 : 321).

7 Nous retenons de cette définition que l'énonciateur de ce "déjà dit » du stéréotype affiche sa socialité, en recourant au discours social. Ce " déjà dit ", inséré dans le texte, est donc»ce qui renvoie à une construction antérieure, extérieure, en tout cas indépendante par opposition à ce qui est « construit » par l'énoncé » (M. Pêcheux 1975 : 88-89).

Cette « construction antérieure » à l'énoncé est préconstruite, et,

Le préconstruit est ce qui fonctionne comme évidence ayant un effet de référence extra-linguistique dans un discours donné. Il n'est porteur de cet effet qu'en tant que sa nature d'élément discursif est occultée, oubliée dans une formation discursive. (...) Cet effet d'immédiate vérité résulte de l'effacement du savoir dans lequel elles [propositions immédiatement vraies] ont été produites. Le préconstruit s'impose au sujet de discours (...). (Fradin et Marandin $1979: 82$ )

9 Ainsi, le stéréotype s'affiche comme une évidence, comme une vérité, et cette "vérité évidente » que l'auteur-voyageur, dans notre cas, utilise ou reproduit comme telle, est une vérité figée.

Dans un article intitulé "Stéréotype, emblème, mythe. Sémiotisation médiatique et figement représentationnel », Henri Boyer (2008) élabore une définition synthétique du stéréotype :

Le stéréotype est (...) considéré comme une sorte de représentation que la notoriété, la fréquence, la simplicité ont imposée comme évidence à l'ensemble d'une communauté (ou d'un groupe à l'intérieur de la communauté). Il s'agit donc d'une structure sociocognitive figée, dont la pertinence pratique en discours est tributaire de son fonctionnement réducteur et univoque et d'une stabilité de contenu rassurante pour les usagers.

$11 \mathrm{Au}$ regard de cette conception, le stéréotype de la nomination ethnique, dans notre corpus, fonctionnerait comme un "catégorisateur», caractérisé par sa dimension générique et son figement sémantique générateurs d'uniformisation des caractéristiques du "Persan » étendues implicitement à tous "les Persans ». Il reste tout de même "une construction imaginaire [qui] ne reflète en rien le réel " (Amossy et Pierrot, 1997 : 36).

Mais, selon Tajfel et Turner (2001: 95),

les représentations stéréotypées sont fonctionnelles même quand elles sont déformées. Il apparaît en effet que si nous avons tendance à accentuer les similitudes entre membres d'un même groupe (l'endogroupe), c'est souvent pour nous valoriser au détriment des autres, de l'exogroupe .

Dans le cas du stéréotype de "l'Oriental » en général et du «Persan » en particulier, nous dirons, pour paraphraser Camilleri et Vinsonneau (1996:50), c'est l'ensemble des 
« images [péjoratives] qu'[une] société projette sur [une autre] à partir de la représentation collective [laudative] stéréotypée qu'elle construit sur son groupe ».

C'est dans cette optique que l'analyse du procédé de l'actualisation/généralisation de la stéréotypie, dans les récits des auteurs-voyageurs que nous avons retenus, prend tout son sens.

14 L'actualisation et la généralisation du stéréotype sont envisagées à travers les occurrences de trois (3) formes génériques du syntagme nominal [article + Persan] : les syntagmes génériques avec les articles définis «le » et «les » et avec l'article indéfini « un ».

Cette option nous a été dictée par les textes de notre corpus dans lesquels ce syntagme nominal est le pivot de la généralisation de la représentation du "Persan », pour l'écriture duquel l'auteur-voyageur adopte systématiquement une attitude normative, généralisante.

En effet, le substantif ethnique "Persan » est précédé quasi-exclusivement d'un de ces trois déterminants génériques ${ }^{1}$.

15 Les trois syntagmes, [le Persan], [Les Persans] et [un Persan], sont tous génériques mais leur généricité n'est pas de même nature: [Le Persan] est un générique massif, [Les Persans] est un générique comptable, et [un Persan] peut être soit générique soit spécifique.

Concernant, la pertinence de cette distinction, nous nous référons à G. Kleiber (1989). Pour les deux premiers déterminants, «le » de « les», il précise ce qui les distingue :

On peut approcher à présent de plus près la différence Les/Le générique en faisant intervenir l'opposition massif/comptable. (...) L'extension d'un $\mathrm{N}$ comptable ou d'un ensemble $\mathrm{N}+$ Modificateur comptable représente une classe générique. Une classe, parce qu'elle regroupe des occurrences discernables (cf. F. Corblin, 1987), et générique, parce que cette classe n'est pas une classe contingente, ou classe fermée, dont on pourrait compter les représentants, mais une classe virtuelle ou classe ouverte (H. Mehlig, 1983), dont les occurrences ne sont pas seulement des occurrences actuelles, mais sont récurrentes dans l'espace et le temps. (...) L'extension d'un $\mathrm{N}$ (ou de $\mathrm{N}+$ Modificateur) intrinsèquement massif (...) est, au contraire, perçue comme homogène: elle rassemble des occurrences non différenciables et ne peut être assimilée par là-même à une classe. (...) Nous postulerons qu'il s'agit d'une entité individuelle constituée par l'ensemble de ses occurrences non discernables. (...) L'individu dénoté par un $\mathrm{N}$ intrinsèquement massif sera un individu générique (...). S'il y avait quelque sens à parler d'un individu " ouvert ", comme il y en a à parler de classe ouverte, on pourrait opposer à la classe ouverte des N comptables l'individu « ouvert » des N massifs. (1989: 81-82)

17 Nous postulons donc, à la suite de G. Kléber, que le syntagme [les Persans] a pour extension une classe générique ouverte (ou virtuelle), constituée de ses occurrences comptables (discernables), et que le syntagme [le Persan] est un individu générique massif constitué de ses occurrences non discernables (non comptables).

Rappelons d'emblée, que dans le contexte de ces récits, la perception de l'altérité nécessite le recours à l'actualisation au sens de passage d'une valeur virtuelle à une valeur réelle, selon $\mathrm{CH}$. Bally $(1932: 136)$ : «actualiser un concept, c'est l'identifier à une représentation réelle du sujet parlant ».

19 Afin d'établir ce rapport de " compréhension » de l'Autre, du/des Persan (s), qu'il veut décrire à ses lecteurs, et que nous appellerons avec Magri-Mourgues des 
" échantillons réels ", l'auteur-voyageur va actualiser les représentations qu'ils en ont et non « la représentation réelle » ou référentielle.

Nous parlerons donc, dans le cas des deux syntagmes génériques, [le Persan]-[les Persans], de « référence virtuelle » au sens de Milner (1978) qui l'oppose à " référence actuelle » : "Le nom en emploi générique ne se confond pas avec l'unité lexicale hors emploi. En effet, il a bien une référence actuelle, comme tout nom en usage; simplement, celle-ci coïncide avec la référence virtuelle, ou plutôt cette dernière, sans être modifiée, est transformée en référence actuelle » (1978: 26-27).

Par cette "référence virtuelle», transformée en "référence actuelle», le nom «Persan-s», en emploi générique, désigne la classe ouverte des Persans (avec «les») ou l'individu massif du Persan (avec «le») et non du/des Persan-s en particulier ou spécifique-s.

Partant du postulat que «le donne la généricité de façon massive, les de façon comptable » (Kleiber, 1989: 76), nous tenterons d'en tirer les conclusions quant à la représentation de l'Autre/Persan-s, en étudiant les occurrences du syntagme de nomination ethnique [le/les/ + Persan-s], dont la distribution dans les cinq (5) récits viatiques est donnée dans le tableau ci-dessous :

\begin{tabular}{|c|c|c|c|}
\hline Récit & $\begin{array}{l}\text { Occurrences } \\
\text { avec » Les » }\end{array}$ & $\begin{array}{l}\text { Occurrences avec } \\
\text { «Le» }\end{array}$ & $\begin{array}{l}\text { Occurrences avec } \\
\text { « un» }\end{array}$ \\
\hline $\begin{array}{l}\text { A. Gardane (De) : Journal d'un voyage } \\
\text {... (1808) }\end{array}$ & 14 & 01 & 04 (spécifiques) \\
\hline $\begin{array}{l}\text { J. J. Morier : Second voyage en Perse ... } \\
\text { (1818) }\end{array}$ & 25 & 02 & $\begin{array}{l}12 \quad \text { (dont } \quad 7 \\
\text { génériques) }\end{array}$ \\
\hline $\begin{array}{l}\text { J M.Tancoigne : Lettres sur la Perse } \\
\text { et... (1819) }\end{array}$ & 33 & 00 & $\begin{array}{ll}02 \quad(\text { dont } & 1 \\
\text { générique }) & \end{array}$ \\
\hline $\begin{array}{l}\text { P. A. E. P. Jaubert : Voyage en Arménie } \\
\text { et... (1821) }\end{array}$ & 38 & 01 & $\begin{array}{l}05 \quad \text { (dont } \quad 2 \\
\text { génériques) }\end{array}$ \\
\hline G.Drouville : Voyage en Perse...(1828) & $47+6$ (au féminin) & 01 & 11 génériques \\
\hline Total & 163 & 05 & $\begin{array}{l}21 \text { génériques sur } \\
34\end{array}$ \\
\hline 189 & $86,24 \%$ & $2,64 \%$ & $11,11 \%$ \\
\hline
\end{tabular}

Dans ce décompte, nous avons inclus les articles contractés au pluriel « des » et « aux », et au singulier « du».

23 Pour la nomination ethnique, l'article « les » est fortement privilégié dans les cinq (5) récits, suivi dans une proportion beaucoup moins importante de l'article générique «un» et dans une proportion nettement plus faible de l'article «le » ${ }^{2}$. Cette tendance des récits viatiques du début du $19^{\text {e }}$ siècle ne fait que prolonger celle, en amont, des récits du $18^{\mathrm{e}}$ siècle : J. Chardin, par exemple, cité par un des auteurs-voyageurs retenus ici, recourt massivement au syntagme générique [les + Persans], dans Les Voyages $d u$ 
chevalier Jean Chardin en Perse et autres lieux de l'Orient (1735): cent vingt-quatre occurrences (en comptant les partitifs), dont quatre-vingt dix-huit avec «les », contre aucune ni avec « le », ni avec « un». En aval, au début du $20^{\mathrm{e}}$ siècle, dans Vers Ispahan (1904) de Pierre Loti, nous dénombrons quinze occurrences du syntagme [les + Persans] dont neuf (9) partitifs. Le texte n'en compte que six (6) en position de sujet, et aucune occurrence ni avec «le», ni avec « un ». L'auteur cible en général des Persans spécifiques.

Pour saisir le sens de cette distribution dans les cinq (5) récits, il nous faut examiner les occurrences des syntagmes [le/les/ + Persan-s] et [un + Persan] dans leurs contextes d'apparition.

\section{L'article défini « les » dans le syntagme générique comptable [les + persans]}

Les auteurs-voyageurs privilégient l'article défini pluriel [les] (86,24\%) pour composer le syntagme de la nomination ethnique, confirmant ainsi que leur vision de l'altérité (Même/Autre) est généralisante et surtout catégorisante. Dans [les Persans], "les » renvoie à la classe des Persans en tant que « collection d'analogues discernables » (Kleiber, 1990 : 58).

Doté du trait comptable, l'article défini pluriel convient à la description des activités collectives de l'Autre que les auteurs-voyageurs veulent décrire à leurs lecteurs. Quelques exemples:

Récit d'A. Gardane (De) :

- Les persans croient que c'est Dariopolis ; d'autres, Hougan. C'est le nom de la contrée. (p.40)

- Les Persans les (chakalls) poursuivent à grands cris (...). (72)

- Les persans s'appliquent un emplâtre de boue (...). (72)

Récit de J. J. Morier :

- Les Persans croient que le voisinage d'un saint communique à ces haillons des qualités particulières contre les maladies. (72)

- Les Persans chassent cet oiseau (...). (301)

- Les Persans plantent le peuplier (...). (121)

Récit de J. M. Tancoigne :

- Les Persans prétendent qu'il (L'Imam Méhdi) est monté aux cieux sans avoir cessé de vivre (...). (162)

- Les Persans peuvent épouser jusqu'à quatre femmes légitimes, et entretenir autant de concubines que leur fortune le leur permet. (20)

- Les Persans comptent encore le temps par les mois alexandrins (...). (33)

Récit de P.A.E.P Jaubert :

- Enfin les Persans croient encore que Tauris est le centre du monde. (156)

- Les Persans s'adonnent au commerce avec ardeur. (282)

- Les Persans ne déploient plus dans leurs parties de chasse cette magnificence dont Chardin a tracé le tableau. Cependant ils ont conservé la chasse au faucon, plaisir dispendieux qui leur vient probablement des Tartares. (346-347)

Récit de G. Drouville : 
- Les Persans aiment passionnément la chasse, aussi les Grands y passent-ils des semaines et même des mois entiers. (32)

- Les Persans se font mutuellement, au Newrouz, des visites et des présents, comme on s'en fait dans nos contrées le premier jour de l'an, et toutes les personnes (...) s'habillent de neuf à cette époque. (33)

- Les Persans, dont je vous ai fait connaître la malpropreté, enveniment encore leurs maux par les remèdes qu'ils emploient pour en guérir. (70)

Comme ici, la grande majorité des occurrences du syntagme [les + Persans] est constituée d'un syntagme nominal, placé à la tête d'une phrase énonciative dont il est le sujet. La totalité des prédicats de ce SN [les Persans] est composée par des prédicats de classe (Galmiche, 1985) et non des prédicats contingents, spécifiques aux contextes particuliers des récits.

Donc, dans ces récits viatiques, « les " s'avère utile pour décliner les traits définitoires de l'Autre dans sa diversité générique comptable, c'est-à-dire en procédant à sa généralisation par catégorisation ${ }^{3}$. Les prédicats sont des propriétés attribuées à tous « les Persans » sous forme d'assertions généralisantes et invariables à la faveur de leurs verbes conjugués au présent de l'indicatif.

Ce sont ou des "prédicats habituels » (avec des verbes d'action au présent - poursuivre, chasser, épouser, planter, envenimer, s'adonner au commerce, se faire des visites, compter, ... -, ou des verbes d'opinion (ou de parole) au présent - croire, prétendre, discuter, etc.), soit des prédicats statifs (avec des verbes d'état au présent), comme dans les exemples suivants :

Récit d'A. Gardane (De) :

- Les persans sont d'excellents cavaliers. (32)

Récit de J. J. Morier :

- Les Persans, qui sont ordinairement assez insensibles aux scènes les plus sublimes et les plus pittoresques de la nature, furent ici frappés d'étonnement et d'admiration. (347)

Récit de J. M. Tancoigne :

- Les Persans sont aussi peu avancés sous le rapport de l'artillerie. On pourrait même dire que cette science si perfectionnée en Europe, leur est totalement inconnue. (76)

Récit de P.A.E.P Jaubert :

- Nous entendons par Orientaux les Turcs, les Arabes et les Persans qui sont comme on sait, sous la même loi religieuse, et gouvernés d'après les mêmes principes. (292-293)

Récit de G. Drouville :

- Les Persans, superstitieux en tout, le sont bien plus encore plus dans ce qui concerne leurs chevaux. (63)

31 Ces prédicats, qu'ils soient habituels (routiniers) ou statifs, sont tous caractérisants : leur syntagme nominal générique sujet, [les Persans], conférant à la phrase son caractère de généricité.

Par le recours pléthorique à l'article défini au pluriel «les", dans les conditions décrites ci-dessus, l'écriture convoque le concret comptable en effaçant en lui toute trace individualisante, distinctive, spécifiante. Cet effacement est d'autant plus explicite que les généralisations des attributs de la catégorie ethnique "Persan » font écho à des textes de référence. Dans la préface de son ouvrage, $G$. Drouville déclare :

Mon intention n'est pas d'écrire une nouvelle histoire de la Perse parce qu'il serait difficile de rien ajouter à ce qu'ont dit à ce sujet Chardin, Thévenot, (...). Plusieurs 
de ces auteurs ont même fourni sur les mœurs et les cérémonies des Persans beaucoup de détails, dont quelques uns offrent assez d'exactitude et de vérité. voyage : « Nous voici en Asie. Dans cette partie du monde, tout est nouveau, la nature et les mœurs. Pour voyager avec fruit, il faudrait avoir dans sa tête l'Histoire sainte et profane, ancienne et moderne » (1808: pp. 2-3). Après lui, P.A.E.P. Jaubert présentait son projet dans l'avant-propos de son récit, avec, également, la référence à des avanttextes :

Afin d'atteindre, autant qu'il m'était possible, ce dernier but [publier une relation qui, en éveillant la curiosité par le récit d'aventures lointaines, puisse également satisfaire cette classe de lecteurs qui demande aux voyageurs des notions positives et précises sur la géographie et l'histoire des pays qu'ils ont visités], je dus recourir aux conseils d'hommes éclairés.

Ainsi, cette représentation catégorisante de l'Autre ne commence pas en faisant tabula rasa d'un "pré-construit », mais elle "re-commence » toujours à partir de lui avec l'objectif de satisfaire les attentes des lecteurs. C'est ce qui explique que dans ces récits, comme dans les autres récits viatiques du même genre, les généralisations des attributs de la catégorie ethnique «Persans » se font écho d'un texte à l'autre et le revendiquent.

Prenons pour illustration deux propriétés de l'ethnotype «Les Persans", dans les cinq récits, mais que l'on retrouve dans la quasi-totalité des récits viatiques qui décrivent "les Persans » jusqu'au début du $19^{\mathrm{e}}$ siècle : [1'hospitalité] et le comportement des Persans vis à vis des [épouses : esclaves, voilées, cloîtrées] :

\begin{tabular}{|c|c|}
\hline [Hospitalité] & [Epouses : esclaves, voilées, cloîtrées] \\
\hline \multicolumn{2}{|l|}{ Récit d'A. De Gardane (1808) } \\
\hline $\begin{array}{l}\text { 1- « Le Cadi et les habitants hospitaliers nous reçoivent } \\
\text { fort bien, ils nous donnent des légumes et des vivres, } \\
\text { sans vouloir accepter de l'argent. » (p. 11) } \\
2 \text { - " Nous avons été dix heures pour aller de Maraç à } \\
\text { Naserabat, pauvre village où nous avons trouvé un toit } \\
\text { hospitalier.» (p. 80) } \\
\text { 3- « Nos manières honnêtes nous les concilient, et ils } \\
\text { nous donnent l'hospitalité dans leurs habitations } \\
\text { souterraines." (p. 21) }\end{array}$ & $\begin{array}{l}11 \text { - «Les Persans regardent avec dédain nos } \\
\text { manières de vivre et de nous habiller; notre } \\
\text { conduite, nos égards envers nos femmes leur font } \\
\text { pitié. Chez eux une femme n'est qu'une esclave." } \\
\text { (p. 57) }\end{array}$ \\
\hline \multicolumn{2}{|l|}{ Récit de J. J. Morier (1818) } \\
\hline $\begin{array}{l}4 \text { - « Le pays où nous entrons était couvert d'Iliâts qui } \\
\text { nous reçurent dans leurs tentes avec beaucoup } \\
\text { d'hospitalité (...).»(p.80) }\end{array}$ & $\begin{array}{l}12 \text { - «Les eunuques abyssiniens sont chargés de la } \\
\text { garde du harem à la Perse ottomane. " (p.210) } \\
13 \text { - «Derrière le Dywan-khânéh est un vaste } \\
\text { anderoun, ou appartement des femmes, que des } \\
\text { portes extrêmement fortes séparent de l'édifice." } \\
\text { (p.377) }\end{array}$ \\
\hline
\end{tabular}




\begin{tabular}{|c|c|}
\hline $\begin{array}{l}5 \text { - «Nous trouvâmes un bon vieillard qui nous reçut } \\
\text { très amicalement; nous fit asseoir à ses côtés, nous fit } \\
\text { servir des pipes et du café (...). » (p.184) }\end{array}$ & $\begin{array}{l}14 \text { - «Plus resserrées que les femmes turques, elles } \\
\text { (les Persanes) ne sortent que le plus rarement } \\
\text { possible, et seulement pour aller visiter leurs } \\
\text { parentes ou leurs amies. Alors, elles (...) sont } \\
\text { couvertes de la tête aux pieds d'une longue pièce de } \\
\text { toile blanche (...); leur visage, leurs mains même, } \\
\text { sont invisibles.» (p.17) } \\
15 \text { - » Le harem du roi de Perse, comme celui du } \\
\text { Grand-Seigneur, est gardé par des eunuques blancs } \\
\text { et des eunuques noirs; mais les uns et ils autres } \\
\text { sont, je crois, moins nombreux et moins puissants } \\
\text { qu'à Constantinople. (...) Les eunuques noirs sont } \\
\text { les seuls qui puissent entrer dans l'intérieur des } \\
\text { appartements des femmes; les blancs sont commis à } \\
\text { la garde des portes extérieures (...). » (p.86) }\end{array}$ \\
\hline \multicolumn{2}{|l|}{ P.A.E.P. Jaubert (1819) } \\
\hline $\begin{array}{l}6 \text { - « Il n'est personne qui ne sache que l'hospitalité est } \\
\text { la vertu que les Musulmans honorent le plus. » (p.310) } \\
\text { - « Ainsi ces scélérats venaient de violer l'hospitalité, } \\
\text { qui leur est prescrite comme la plus sainte des lois } \\
\text { (...). » (p.40) } \\
7 \text { - « Les peuples qui se livrent le plus au vol et au } \\
\text { brigandage sont souvent aussi ceux qui remplissent le } \\
\text { plus rigoureusement les devoirs de l'hospitalité; et (je } \\
\text { le dis à regret) c'est ce qui fait que, dans l'orient, un } \\
\text { voyageur expérimenté redoute surtout les contrées où } \\
\text { cette vertu est le plus en honneur (...). » (p.83) }\end{array}$ & $\begin{array}{l}16 \text { - «Les femmes, on ne le sait que trop, sont, dans } \\
\text { ce pays les premières esclaves de leur mari. » (p. } \\
\text { 298) } \\
\text { - " Les femmes sont tellement exclues de la vie des } \\
\text { hommes qu'il n'est ni permis ni décent d'en } \\
\text { prononcer le nom. » (p.300) } \\
17 \text { - " Les Persanes négligent extrêmement leur } \\
\text { parure dans l'intérieur du harem. (..) Hors de leurs } \\
\text { maisons, elles ont, comme on le sait, la tête couverte } \\
\text { et un voile blanc qui leur tombe jusqu'aux pieds. " } \\
\text { (p.322) } \\
18 \text { - "Les tentes du harem sont peu éloignées du } \\
\text { divan-khânéh. On assure qu'elles le surpassent de } \\
\text { beaucoup en magnificence. La garde en est confiée à } \\
\text { un certain nombre d'eunuques dont la surveillance } \\
\text { est plus rigoureuse encore qu'en Turquie. ." } \\
\text { (pp. 343-344) }\end{array}$ \\
\hline
\end{tabular}


8 - «(...) La province (Ourouméa) n'en est pas moins citée aujourd'hui comme une des plus nobles, des plus braves et des plus hospitalières de la Perse.

Cette dernière vertu est si scrupuleusement exercée en Perse, qu'il serait rare de rencontrer un individu quelconque, à l'entrée d'une ville ou d'un village, qui n'accueillit pas un étranger par ces mots: Meneumkonack (vous êtes mon convive). Ces mots ne sont pas vides de sens, comme les autres politesses usitées en Perse, puisque celui qui les prononce se considère grièvement offensé si on le refuse. » (p. 60)

9 - «Les Persans sont (...) d'une hospitalité sans exemple (...) (pp. 45-46).

10 - Les Persans sont très hospitaliers (...). » (p.116)
19 - «Le roi a deux enceintes pareilles, l'une entoure le divan et l'autre le harem; un mur soigneusement fermé les sépare (...). » (p.153)

20 - "J'ai déjà dit que l'extérieur était gardé par quatre mille hommes, et l'intérieur par les eunuques de service qui veillent toute la nuit autour des tentes du harem. » (p.155)

21 - Les dames ne sortent cependant jamais que couvertes de chaderas blancs qui leur enveloppent tout le corps, et comme elles ne font pas usage du roubend pour cacher leur figure, elles lui substituent des voiles non moins ridicules. » (p.182)

Dans les citations 9, 10 et 11, le syntagme nominal [les Persans] est un syntagme générique parce qu'il satisfait aux deux critères définis par Kleiber et Lazzaro : en tant que tel, il renvoie à la classe ouverte (voir supra) des Persans " répondant au double critère de constitution suivant: le détachement par rapport à l'existence hic et nunc de ses membres et la récurrence dans le temps » (1987:94):

9 - Les Persans sont (...) d'une hospitalité sans exemple (...),

10 - Les Persans sont très hospitaliers (...),

11 - Les Persans regardent avec dédain nos manières de vivre et de nous habiller ; notre conduite, nos égards envers nos femmes leur font pitié. Chez eux [les Persans] une femme n'est qu'une esclave.

Le premier critère, à savoir "le détachement par rapport à l'existence hic et nunc de ses membres » et le second, à savoir " la récurrence dans le temps", sont attestés à la fois :

- d'une façon interne à l'énoncé - le syntagme générique [les persans] conférant la généricité au prédicat statif dont il est le sujet ;

- d'une façon intratextuelle, par la répétition dans le même texte, de la même idée à travers des extensions syntaxico-sémantiques quasi identiques de [les Persans] et de ses synecdoques dans les autres exemples relatifs à ce thème (le Cadi et les habitants, les Iliâts, un bon vieillard, les Musulmans, la Perse, etc.) ;

- d'une façon intertextuelle, par sa répétition et celle de ses synecdoques d'un texte à l'autre, et enfin,

- d'une façon externe au texte par indexation du discours de l'auteur-narrateur à des "éléments doxiques ${ }^{4}$ sur [les Persans] qui achèvent de situer l'énoncé (SN + SV) hors du temps : ex. 6 :» Il n'est personne qui ne sache que l'hospitalité... »; ex. 16: «Les fermmes, on ne le sait que trop... »; ex. 17 : « Hors de leurs maisons, elles ont, comme on le sait,... ».

Ces exemples montrent le processus de coalescence lexicalisée du syntagme nominal [les Persans] avec ses propriétés, [hospitalité] et [esclavage et claustration des épouses] : les prédicats [hospitalier] et [esclavage /claustration des épouses] se donnent à lire comme des propriétés permanentes, intrinsèques des Persans, au point qu'elles peuvent accueillir l'ambivalence comme dans l'exemple $\mathrm{N}^{\circ} 7$ ou dans ceux-ci :

- «Je croyais toutes les femmes malheureuses en Turquie et en Perse, mais dans un sérail il y a de beaux jardins ... » (A. Gardane (De), 1808 : 89) ; 
- «On y voit souvent des épouses éplorées, à genoux près du tombeau d'un époux, preuve certaine que les Orientaux ne traitent pas leurs femmes aussi mal qu'on le croit communément » (P.A.E.A.P. Jaubert, 1819 : 304-305).

Cette remise en cause des croyances admises (" je croyais ...mais », « on a souvent des idées ... fausses »), au lieu de restreindre la portée des phrases génériques, ne fait au contraire, que la conforter en l'installant comme une totalité dont on peut débattre.

Nous pouvons étendre notre démonstration à une multitude d'autres propriétés qui, par le truchement du même procédé, sont associées au syntagme générique [les Persans] : ([Musulmans], [excellents cavaliers], [superstitieux], [défiants vis à vis des chrétiens et des juifs], [aimant la chasse au faucon], etc.

41 Ces récits, en effet, représentent l'Autre, le Persan, à travers les mêmes propriétés, appréhendées sous le même angle de vue, pour les principales d'entre elles. Ce sont surtout celles relevant de l'habitus persan décrit de manière identique à travers des synecdoques de [Persans]: leur nourriture, leur façon de se comporter avec les étrangers, avec les hommes d'autres religions (les « chrétiens » et les « juifs»), avec les femmes, avec les chevaux et les autres animaux sauvages et domestiques, leur manière de parler leur langue, de (mal) faire la guerre par rapport aux Européens, etc.

Ces généralisations, introduites par l'article défini au pluriel, « les », et qui se répètent d'un texte à l'autre, aboutissent à une «essentialisation» telle que la conçoit J. Maisonneuve (1989) cité par R. Amossy et al. (1997: 51) :

Le propre de la stéréotypie, c'est d'être grossière, brutale, rigide et de reposer sur une sorte d'essentialisation simpliste où la généralisation porte à la fois : - sur l'extension : attribution des mêmes traits à tous les êtres ou objets désignables par un même mot (...) - sur la compréhension : par simplification extrême des traits exprimables par des mots.

43 Ainsi, la généralisation par "extension» et «simplification» des attributs (ou propriétés) caractéristiques des Persans, les représente d'une façon homogène, schématisée, "décontextualisée ", "des-historicisée ». De ce fait, en se répétant d'un texte à un autre, dans les conditions identiques de réception - ces textes partageant le même lectorat, donc le même horizon d'attente -, ce qui était attribut se transforme en essence ethnotypique, faisant du [Persan] une abstraction.

44 L'auteur-voyageur adopte à l'égard de l'Autre une attitude normative, évinçant sa spécificité individualisante ancrée dans le temps et l'histoire, pour une classe homogène non spécifique, à référence virtuelle. Un des moyens pour y parvenir est le recours à l'article défini « les » dans la constitution du syntagme générique comptable [les Persans].

45 Le second est le recours, mais dans une moindre mesure, à l'article défini au singulier «le», pour la constitution d'un syntagme générique massif [le Persan] et au syntagme générique [un Persan].

\section{Le syntagme générique [le + persan] et le syntagme mixte [un + persan]}

Les récits viatiques de notre corpus n'utilisent que très faiblement l'article défini au singulier, « le » dans le syntagme générique massif [le +Persan] (2,64 \%) :

Récit d'A. Gardane (De) : 
1 - Le persan ne sort pas de sa ville. Les grands qui vont quelquefois d'une province dans l'autre portent leurs tentes; mais les étrangers sont toujours au caravansérail. (82)

Récit de J. J. Morier :

2 - Il n'y a au monde aucun peuple aussi hâbleur que le Persan; c'est là l'essence de son caractère: aucune nation n'est aussi perfide. C'est souvent même au moment où il vous accable de politesses qu'il faut s'en méfier. (...) (227-228 : notes de bas de page)

Récit de J. M. Tancoigne : $\varnothing$

Récit de P.A.E.P Jaubert :

4 -Un turk exaltera la grandeur, la magnificence ottomane. (...) Le Persan vantera la beauté, la fertilité des jardins du Chirâz, les fruits délicieux de Yezd et les monuments d'Ispahan, ville qu'il appelle encore avec emphase noussfi djehân, moitié de l'univers. (319)

Récit de G. Drouville :

5 - Le caractère du Persan est peut-être le plus heureux et le plus doux de toutes les nations d'Orient; et s'il differe un peu de province en province, ce n'est qu'en raison du grand nombre d'étrangers que cette nation (...) fut obligée d'adopter (...). (43)

47 Ces énoncés montrent que le syntagme générique de la nomination ethnique avec l'article défini au singulier, [le Persan], est, comme le précédent, en position de sujet, mais il est exclusivement réservé à des idées reçues sur «le Persan ", des "évidences partagées » (cf. « éléments doxiques ») avec les lecteurs.

48 [Le Persan] est bien un syntagme générique massif en ce qu'il fait du »Persan » un « individu générique " (Kleiber, 1989: 79) qui ne comporte pas d'ancrage temporel, qui ferait du réferent un exemplaire (occurrence ou sous-classe ». En effet, «les sous-parties qui le composent [les différents prédicats] (...) récurrent dans le temps et l'espace. (Kleiber, op.cit. : 82).

De plus les prédicats de ce syntagme, exclusifs (ne sort pas), ou exclusifs et superlatifs (le plus/aucun peuple au monde), confortent le non-ancrage de [le Persan] dans l'espacetemps conjoncturel.

50 A la différence de «les» de «les Persans », l'article défini singulier «le» de «le Persan» écarte donc toute référence à la diversité comptable. Il introduit un substantif massif dans son abstraction (Kleiber, 1990 : 37). C'est ce qui fait de lui non seulement un indicateur de la généricité mais aussi celui de la stéréotypie par excellence. A la faveur de cette massification, la nomination ethnique désigne [le Persan] comme hyperonyme de tout "Persan » et de " tous les Persans ».

51 Mais tous deux, "le» générique massif, comme "les» générique comptable, concourent tous deux à l'abstraction de l'ethnotype «Persan». Cependant, par sa puissance d'abstraction, «le» frappe d'inanité le voyage à la découverte de l'Autre, décevant ainsi les attentes des lecteurs. C'est sans aucun doute, la raison pour laquelle, [le Persan] n'est que rarement utilisé dans les récits viatiques de notre corpus.

52 Le recours à l'article indéfini « un », dans la composition du syntagme [un Persan], est relativement plus important $(11,11 \%)$ que celui avec «le ", même s'il est très loin de celui avec « les». Cela s'explique par la possibilité qu'il a d'exprimer soit le générique, soit le spécifique.

53 Reprenons, pour distinguer les deux emplois de cet article indéfini, la proposition de J. Hawkins cité par M. Galmiche (1985: 24) :

La différence entre un indéfini générique et un indéfini spécifique est (...)

fondamentalement pragmatique ; elle dépend du fait de savoir si le contexte amène 
l'auditeur à croire que le locuteur a ou bien n'a pas de réfèrent particulier à

l'esprit . Persan] diffère de celui avec l'article défini « les » du syntagme [les Persans], même s'ils renvoient tous deux à une classe ouverte (virtuelle par rapport au contexte de l'énoncé et de son énonciation) : [les Persans], générique comptable, convient à la description des Persans à travers des prédicats statifs ou habituels, tandis qu'[un Persan] est plutôt réservé à la façon qu'auraient les Persans de se conduire par rapport à des principes et à des préceptes, comme par exemple dans :

- Dans le fait, un Persan préfère le pouvoir à l'argent. (Morier, 76)

- (...) il (le mihmândâr) accompagna ces mots de tous les termes, de tous les lieux communs de mépris pour la famille du gouverneur ; pour son père, pour sa mère, pour son épouse, pour ses filles, etc., etc., qui sont toujours les premiers objets sur lesquels retombe un Persan dans sa fureur. (Morier, 224-225)

- Un Persan ne se ruine jamais qu'en promesses. (Morier, 228)

- En pays étranger, le plus clairvoyant est aveugle (Proverbe arabe : note de bas de page), et garde-toi de croire un Persan, lors même qu'il te dira la vérité. (Jaubert, 34)

- Un Persan peut rester depuis le matin jusqu'au soir, assis sur les talons, près d'une fenêtre, à regarder le jet d'eau qui est au-dessous de lui, (...). (Drouville, 96)

- En quelque lieu que se trouve un Persan, quelles que soient ses occupations, il quitte tout au moment de la prière (...). (168)

56 Pour M. Galmiche (1985: 31), « les syntagmes du type UN N entrent tout naturellement dans les jugements dits "déontiques", c'est-à-dire les propositions destinées à véhiculer une règle d'ordre comportemental ou moral ».

$57 \mathrm{Au}$ regard des récits de notre corpus qui usent de ce générique, ces «jugements dits déontiques " participent des propriétés intrinsèques, génériques, des Persans et donc de leur stéréotypisation.

$58 \mathrm{Au}$ cours de cette analyse relativement sommaire, nous avons traité des actualisateurs de la généralisation et de la généricité, en tant qu'elles participent de la façon la plus manifeste au processus de la stéréotypie dans la nomination ethnique.

Nous avons essayé d'expliquer la pertinence du recours à chacune des trois formes génériques - [le Persan], [les Persan] et [un Persan] - dans les cinq récits de notre corpus.

60 C'est ainsi que leurs auteurs ont géré l'étape la plus importante de la saisie d'un univers autre qui est celle de la neutralisation des différences qui mène à la généralisation, ou de l'obtention du général à partir du particulier en usant de la figure de la synecdoque. Celle-ci implique qu'une donnée représentative soit employée pour parler de l'ensemble dont elle fait partie.

61 La dichotomie qui existe entre le singulier et le pluriel est parallèle à celle du passage du particulier au générique. Dans toutes les occurrences relevées, ladite stratégie va de pair avec celle qui passe du concret à l'abstrait.

62 Cette saisie descriptive, générale et abstraite, a le défaut d'être grossière, incomplète et donne lieu à une représentation non conforme au référent réel. 


\section{BIBLIOGRAPHIE}

AMOSSY R., Les idées reçues : sémiologie du stéréotype, Nathan, Paris, 1999.

AMOSSY R., HERSCHBERG PIERROT A., Stéréotypes et Clichées, langue discours société, Nathan, 1997.

AMOSSY R., L'Argumentation dans le discours, Armand Colin, Paris, 2006.

BALLY C., Linguistique générale et linguistique français, A. Francke, a.g. Verlag, Berne, 1932.

BOYER H., «Stéréotype, emblème, mythe. Sémiotisation médiatique et figement

représentationnel », in Mots, les langages du politique, $N^{\circ}$ 88, ENS Éditions, 2008, pp. 99-113. Mis en ligne le $01 / 11 / 2010$. Consulté sur le site : https://mots.revues.org/14433

CAMILLERI C., VINSONNEAU G., Psychologie et culture : Concepts et Méthodes, Colin, Paris, 1996.

CHARDIN J., Les Voyages du chevalier Jean Chardin en Perse et autres lieux de l'Orient, Amsterdam, Aux dépens de la Compagnie, 1735. Consulté sur le site :

https://archive.org/stream/voyagesducheval04lenogoog/voyagesducheval04lenogoog_djvu.txt CORBLIN F., Indéfini, défini, démonstratif, Droz, Genève, 1990.

DETRIE C., SIBLOT P., VERINE B., Termes et concepts pour l'analyse du discours. Une approche praxématique, Champion, Paris, 2001.

FRADIN B., « Anaphorisation et stéréotypes nominaux », in Lingua, N 64, 1984, pp. 325-369.

FRADIN B., MARANDIN J.M., « Autour de la définition : de la lexicographie à la sémantique », in Langue française, Volume 43, $\mathrm{n}^{\circ}$ 1, 1979, pp. 60-83.

GALMICHE M., "Phrases, syntagmes et articles génériques », in Langages, n 79, Générique et généricité, Larousse, 1985, pp. 2-39. Disponible sur le site : http://www.persee.fr/doc/ lgge_0458726x_1985_num79_2468

KLEIBER, G. et LAZZARO, H., « Qu'est-ce qu'un syntagme nominal générique ? ou les carottes qui poussent ici sont plus grosses que les autres », dans Rencontre(s) avec la généricité, Klincksieck, Paris, 1987, pp. 73-111.

KLEIBER G., « Généricité et typicalité », in Le Français Moderne, N 57, 1989, pp. 127-154.

KLEIBER G., L’Article « le » générique. La généricité sur le mode massif, Droz, Genève, 1990.

LOTI P., Vers Ispahan, Paris, Calmann-Lévy Editeurs, 1904.

Consultable sur les sites :

http://gallica.bnf.fr/ark:/12148/bpt6k5809943k.r=.langFR

http://www.bibebook.com/bib/vers-ispahan

MAGRI-MOURGUES V., « Détermination nominale et extension référentielle : la construction du stéréotype dans le récit de voyage », in Le Français Moderne, Revue de linguistique Française, $\mathrm{n}^{\circ}$ 1, CILF, 2005, pp. 59-74.

MAGRI-MOURGUES V., «La description dans le récit de voyage », in Cahiers de narratologie CIRCPLES, Nice, $N^{\circ} 7,1996$, pp. 5-14.

MILNER J.-C., De la syntaxe à l'interprétation, Seuil, Paris, 1978. 
PECHEUX M., Les vérités de La Palice, Ed. F. Maspéro, 1975, pp. 88-89.

TAJFEL H., TURNER, J., An integrative theory of intergroup conflict. Intergroup relations : Essential readings, NY, US : Psychology Press, New York, 2001, pp. 94-109.

DROUVILLE Gaspard, Voyage en Perse fait en 1812-1813, Pochard, Paris, 1828. Disponible sur les sites :

https://archive.org/stream/gri_voyageenpers02drou/gri_voyageenpers02drou_djvu.txt https://books.google.dz/books ?id =PfFaAAAAQAAJ

GARDANE Ange (De), Journal d'un voyage dans la Turquie d'Asie et la Perse, Le Normand, Paris, 1808. Disponible sur les sites :

https://books.google.dz/books ?id =vJZGAQAAMAAJ

https://archive.org/stream/bub_gb_4hIZgjrAOGUC/bub_gb_4hIZgjrAOGUC_djvu.txt

JAUBERT Pierre-Amédée-Émilien-Probe, Voyage en Arménie et en Perse, effectué en 1805-1806 accompagné par Notice sur le Ghilan et le Mazandéran de Camille-Alphonse Trézel, Pélicier et Nepveu, Paris, 1821. Disponible sur le site : https://books.google.fr/books ?id =aJAbsZ-

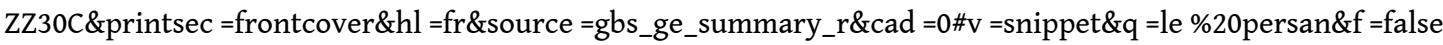

MORIER Jacques, Second voyage en Perse, en Arménie et dans l'Asie-mineure fait de 1810 à 1816, Gide

Fils, Paris, 1818. Disponible sur le site : https://books.google.dz/books ?id =YQsPAAAAQAAJ

TANCOIGNE J.M., Lettres sur la Perse et la Turquie d'Asie, Nepveu, Paris, 1819. Disponible sur le site :

https://books.google.dz/books ?id =lGYOAAAAQAAJ

\section{NOTES}

1. Dans les cinq récits, les emplois d'autres déterminants sont très rares et ne sont utilisés que dans des cas spécifiques au contexte narratif particulier du récit considéré: l'adjectif démonstratif « ce » ([ce Persan] : 2 occurrences), l'adjectif possessif « nos » ([nos Persans]) : trois occurrences, l'adjectif de quantité «plusieurs» ([plusieurs Persans]) : deux occurrences. Ils ne sont donc pas génériques et de ce fait ne contribuent pas directement au processus de stéréotypisation auquel nous nous intéressons ici. C'est la raison pour laquelle nous ne les avons pas étudiés.

2. Nous n'avons pas pris en considération le syntagme « le persan » quand il s'agissait de désigner la langue.

3. Selon G. Kleiber (1990:12), la catégorie est « cette opération mentale qui consiste à ranger ensemble des choses différentes [qui] se retrouve dans toutes nos activités de pensée, de perception, de parole, dans nos actions aussi »).

4. Nous nous en remettons à R. Amossy qui préconise de parler dans certains cas d'« éléments doxiques" plutôt que de doxa: "Nous utiliserons le terme d'interdiscours pour renvoyer à la dissémination et à la circulation des éléments doxiques dans des discours de tous types. Dans la mesure où il s'agit de déceler l'inscription ponctuelle dans le discours oral ou écrit des évidences partagées ou des plausibilités d'une collectivité datée, on parlera d'éléments doxiques plutôt que de doxa. Les notions d'élément doxique et d'interdiscours permettent ainsi de marquer à quel point l'échange argumentatif est tributaire d'un savoir partagé et d'un espace discursif, tout en évitant de conférer à ces matériaux préexistants une trop grande systématicité. » (R. Amossy, 2006 : 110). 


\section{RÉSUMÉS}

Le récit viatique, surtout au $19^{\mathrm{e}}$ siècle, est fondé sur la dialectique du Même (en l'occurrence l'«Occident ») et de l'Autre (l'« Orient»). Au regard des nombreuses études sur le sujet, il est admis aujourd'hui que ce type de récit se définit comme un espace de (re)production de clichés et de topoi qui associent le nouveau au déjà-dit doxique, de manière à satisfaire l'horizon d'attente de ses lecteurs. C'est à l'étude de quelques éléments actualisateurs de la stéréotypie, dans ce parangon de la représentation généralisée et homogénéisée de l'Autre et de son univers ethnique, qu'est consacré le présent article. Nous étudions l'écriture de l'altérité en nous concentrant sur le traitement des actualisateurs généralisants, notamment les déterminants définis « les » et « le » du syntagme générique de la nomination ethnique, [les/le Persan-s], dans cinq (5) récits de voyageurs français du début du $19^{\mathrm{e}}$ siècle : A. Gardane (De) (1808), J. J. Morier (1818), J. M. Tancoigne (1819), P. A. E. P. Jaubert (1821) et G. Drouville (1828).

The travel literature, especially in the nineteenth century, is based on the dialectic of the Same (in this case the "West") and the Other (the "East"). From the point of view of the numerous studies on the subject, it is now accepted that this type of literature is defined as a space of (re) production of clichés and topoi that associate the new with the doxic already-said, in order to satisfy the horizon of expectation of its readers. It is with the study of some actualizer elements of the stereotypy in this paragon of the generalized and homogenized representation of the Other and its ethnic universe that this article is devoted. We study the linguistic means of the writing of otherness by focusing on the treatment of generalizing actualizers, in particular the definite article "the" (in its singular and plural form), of the ethnic nomination [the Persan-s] in five (5) texts of French travelers from the early 19th century: C.M. Gardane (1808), J. Morier (1818), J.M. Tancoigne (1819), P.A. É. P. Jaubert (1821) and G. Drouville (1828).

\section{INDEX}

$$
\text { التصوير النمطي, أدب الرحلة, غيرية, مُحيّن عام, تعيين إثني, تحديدفهرس الكلمات المفتاحية: }
$$

Keywords : stereotypy, travel literature, otherness, generalizing actualizers, ethnic nomination, essentialization, the Persian ethnotype, Travel in Persia in 1812-1813, Journal of a trip to the Asian Turkey and Persia, Travel to Armenia and Persia in 1805-1806, Second voyage to Persia Armenia and Asia Minor from 1810 to 1816, Letters on Persia and Asian Turkey, Drouville G., Gardane C.M. (Count of), Jaubert P. A. P., Morier J., Tancoigne J. M.

Mots-clés : Stéréotypie, récit de voyage, altérité, actualisateur généralisant, nomination ethnique, essentialisation, l'ethnotype Persan, Voyage en Perse fait en 1812-1813, Journal d'un voyage dans la Turquie d'Asie et la Perse, Voyage en Arménie et en Perse effectué en 1805-1806, Second voyage en Perse en Arménie et dans l'Asie-mineure fait de 1810 à 1816, Lettres sur la Perse et la Turquie d'Asie, Drouville G., Gardane A. (De), Jaubert P. A. E. P., Morier J. J., Tancoigne J. M. 


\section{AUTEURS}

\section{AFSANEH POURMAZAHERI}

Université de Téhéran Téhéran - Iran 